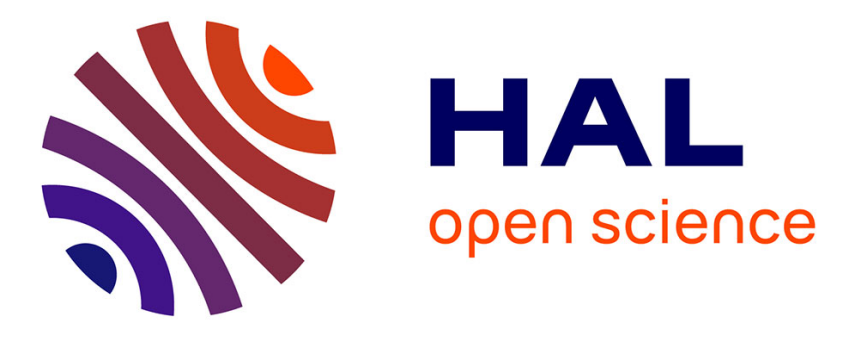

\title{
Changing the color of textiles with realistic visual rendering
}

\author{
Mathieu Hébert, Lambert Henckens, Justine Barbier, Lucie Leboulleux, \\ Marine Page, Lucie Roujas, Anthony Cazier
}

\section{- To cite this version:}

Mathieu Hébert, Lambert Henckens, Justine Barbier, Lucie Leboulleux, Marine Page, et al.. Changing the color of textiles with realistic visual rendering. Measuring, Modeling, and Reproducing Material Appearance 2015, SPIE/IS\&T, Feb 2015, San Francisco, United States. pp.93980Q. hal-01160298

\section{HAL Id: hal-01160298 \\ https://hal.science/hal-01160298}

Submitted on 5 Jun 2015

HAL is a multi-disciplinary open access archive for the deposit and dissemination of scientific research documents, whether they are published or not. The documents may come from teaching and research institutions in France or abroad, or from public or private research centers.
L'archive ouverte pluridisciplinaire HAL, est destinée au dépôt et à la diffusion de documents scientifiques de niveau recherche, publiés ou non, émanant des établissements d'enseignement et de recherche français ou étrangers, des laboratoires publics ou privés. 


\title{
Changing the color of textiles with realistic visual rendering
}

\author{
Mathieu Hébert ${ }^{a, b}$, Lambert Henckens ${ }^{c}$, Justine Barbier $^{b}$, Lucie Leboulleux ${ }^{b}$, Marine Page ${ }^{b}$, Lucie \\ Roujas $^{b}$, Anthony Cazier ${ }^{a, b}$ \\ ${ }^{a}$ Université de Lyon, Université Jean Monnet de Saint-Etienne, CNRS UMR5516 Laboratoire \\ Hubert Curien, 18 rue Benoît Lauras, F-42000 Saint-Etienne, France. ${ }^{b}$ Institut d'Optique, 18 rue \\ Benoît Lauras, F-42000 Saint-Etienne, France. ${ }^{C}$ Lembart S.A.S., L'Imprimerie, Lot B18, 5 rue \\ Javelin Pagnon, F-42000 Saint-Etienne, France.
}

\begin{abstract}
Fast and easy preview of a fabric without having to produce samples would be very profitable for textile designers, but remains a technological challenge. As a first step towards this objective, we study the possibility of making images of a real sample, and changing virtually the colors of its yarns while preserving the shine and shadow texture. We consider two types of fabrics: Jacquard weave fabrics made of polyester warp and weft yarns of different colors, and satin ribbons made of polyester and metallic yarns. For the Jacquard fabric, we make a color picture with a scanner on a sample in which the yarns have contrasted colors, threshold this image in order to distinguish the pixels corresponding to each yarn, and accordingly modify their hue and chroma values. This method is simple to operate but do not enable to simulate the angle-dependent shine. A second method, tested on the satin ribbon made of black polyester and achromatic metallic yarns, is based on polarized imaging. We analyze the polarization state of the reflected light which is different for dielectric and metallic materials illuminated by polarized light. We then add a fixed color value to the pixels representing the polyester yarns and modify the hue and chroma of the pixels representing the metallic yarns. This was performed for many incident angles of light, in order to render the twinkling effect displayed by these ribbons. We could verify through a few samples that the simulated previews reproduce real pictures with visually acceptable accuracy.
\end{abstract}

Keywords: Textile, color imaging, polarized imaging, angular reflectance, visual rendering.

\section{INTRODUCTION}

Everyone knows the variety of appearances of fabrics that can be obtained according to the selected fibers, the structure of the yarns, their preparation by spinning, coating and dyeing, the weaving and the finishing... Infinite variations of color, shine, glaze, transparency and texture may be obtained. Their visual rendering is therefore difficult to predict from these numerous parameters, and only the production of samples allows visualizing the desired rendering. However, producing samples is often an expensive and time-consuming constraint for professionals. A realistic simulator of fabrics where parameters could be virtually modified would represent a high benefit. This is especially true in applications where the fabric is integrated into a global multi-material design and thus needs to satisfy precise rendering constraints.
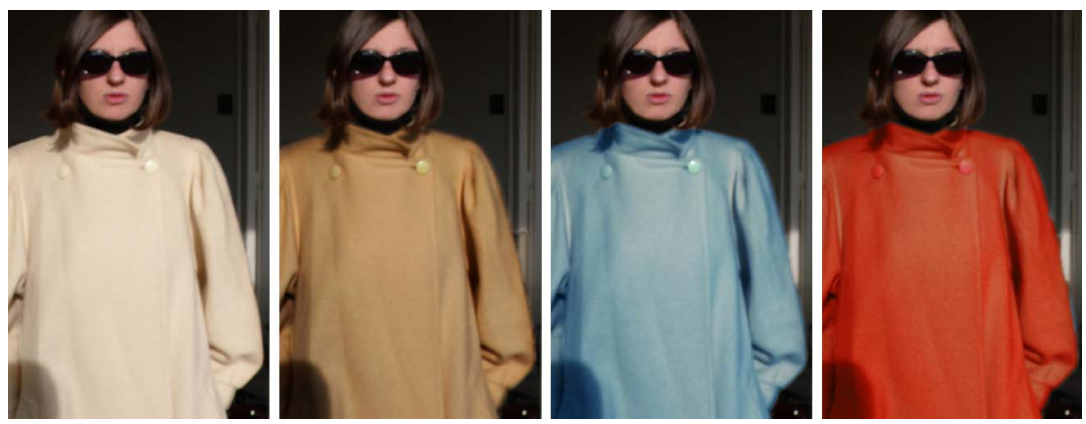

Figure 1. Showing a number of colors for a same fabric would be highly profitable for textile designers, but remains a technological challenge since shadow and gloss texture is crucial for realistic visual rendering. This example was realized by transforming the original image, on the left, with Adobe Photoshop. 
Fig. 1 shows an example of image where an article of clothing is digitally recolored with different tones. The coloration was performed with Adobe Photoshop by selecting the area occupied by the fabric in the image, then changing the hue and chroma thanks to the Hue/Saturation tools. The rendering is acceptable because the fabric is continuous, uniform, and strongly diffusing, almost Lambertian. Fig. 2, however, illustrates the difficulties of recoloration that arises with more complex textiles integrated in packaging designs, for example embroidered openwork lace or black and gold Jacquard weave. The top image is the original one, and the bottom image the result of coloration operations again performed with Adobe Photoshop. On the left bottle, the black lace is changed to red by using the Selective Color tool and modifying the black tone. Only the yarns are recolored while their shadow on the bottle and the bottle itself are almost unchanged; however, the color of the yarns is not realistic at all due to the absence of gloss and shadow texture which is expected for a fabric of non-black yarns. On the bottle in the middle, the blue lace was changed to green by using the Hue/Saturation tool, and the embroideries were changed to purple by selecting them manually and using again the Hue/Saturation tool. Many artifacts are visible when zooming on the image. Moreover, the white embroidery at the center of the bottle is almost impossible to select, even manually; its color was therefore not changed. For the bottle of the right, the black and gold colors where changed by using the Selective Color tool and modifying the black, respectively the white tones. The blue colors on the two faces of the bottle are two close from each other, and the cyan metal yarns do not give an impression of metallic reflection. This shows how challenging is the production of realistic recoloration from digital images.
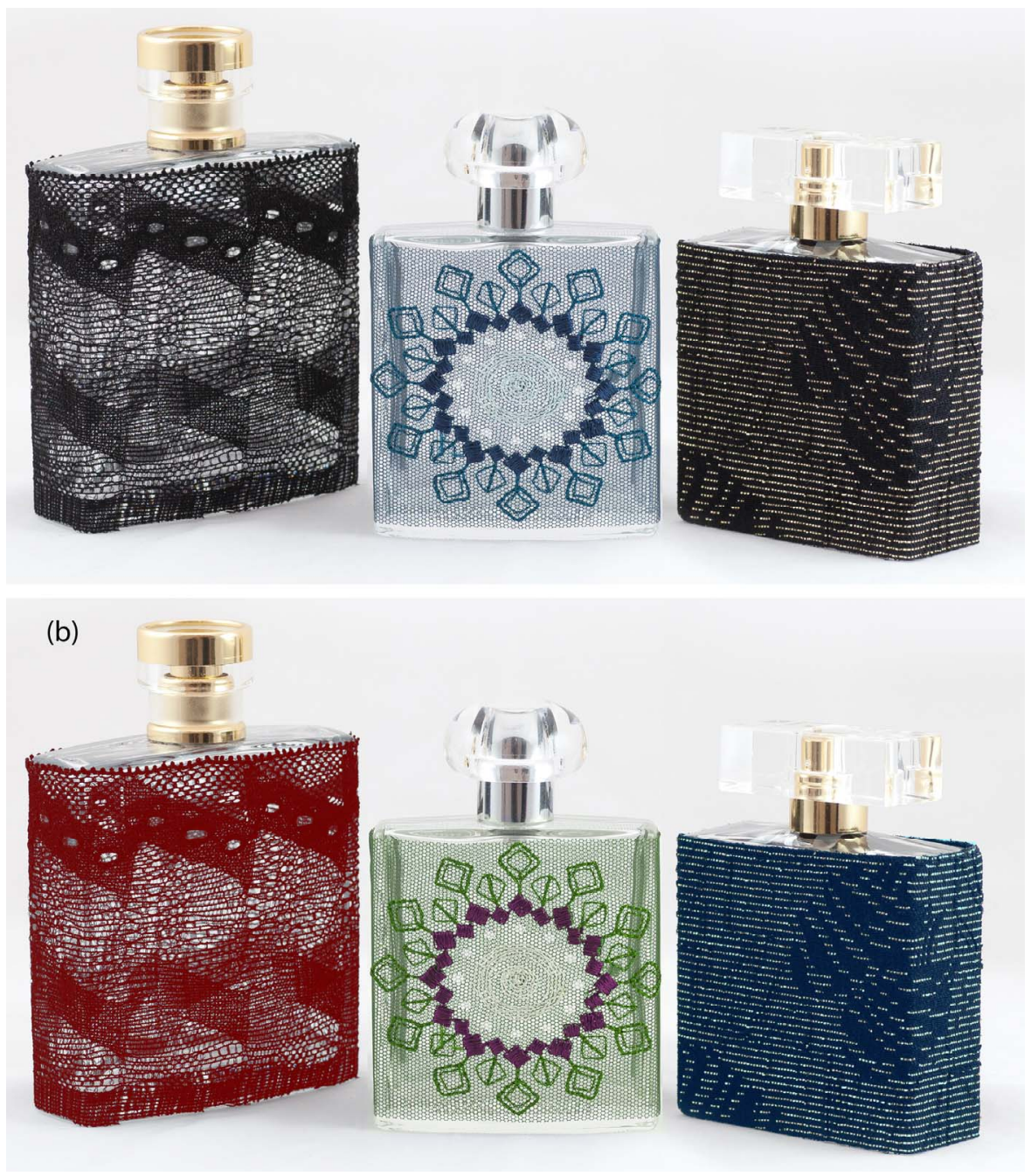

Figure 2. (a) Original and (b) digitally recolored images of bottle covered by pieces of embroidered laces or Jackard fabrics. Courtesy Orimono company. 
Accurate simulation of the visual rendering of fabric surfaces in a given scene requires advanced skills in image synthesis in order to incorporate the complex geometry of illumination, the 3D shape of the object, the angular and spectral/color reflectance of the material, and the position of the observer in the scene. One key point in this chain, on which we focus in this paper, is the reflectance of the fabric: the local irradiance on the surface and the radiance reflected towards the observer can then be deduced from it. Various works intended to predict the spectral reflectance of blends of colored yarns in order to ease the dye formulation for reproducing a target color. Physical equations based on the Stearns-Noechel ${ }^{1-3}$ or the Kubelka-Munk ${ }^{4-7}$ theories are used and improved in order to model the absorption and scattering of light by the different pre-colored fibres. They generally rely on a prior knowledge of the spectral reflectance of the individual fibres and their respective quantities in the blend. Mathur et al. ${ }^{7}$ recently predicted with promising accuracy the color of Jacquard weavings from individual color of yarns and Jacquard weaving patterns under diffuse illumination. However, the spectral reflectance averaged over a large area is not sufficient for realistic visual rendering of most fabrics because of their angle-dependent reflectance properties, especially in the case of satins and Jacquard weavings whose gloss and shadow texture have a noticeable influence on the global appearance. As far as we could see, no prediction method explicitly incorporates the problem of textured reflectance, even by simple modelling. The 3D structure model for textiles proposed by Shidanshidi et al..$^{8-9}$ in order to study their mechanical and physical properties might help to preview some angle-dependent shine effects according to the illumination and observation conditions in addition to the color rendering, but it seems that this area has not been explored yet. In this study, we investigate the possibility to simulate what an existing woven fabric would look like with different yarn tones while preserving the specular reflections at the surface of the yarns and the shadows between them.

The methods that we propose are based on high resolution color images of a real piece of fabric, which show the texture as viewed at a short distance under the illumination and observation conditions used in the measurement. We assume that undersampling the images is sufficient to simulate the appearance of the fabric from a larger distance under the same illumination and observation geometry. Two different methods are proposed to identify in the image the respective positions of the different yarns, i.e., the weft and warp yarns defined in Figure 2. This enables to apply the color changing operations on each of them separately.

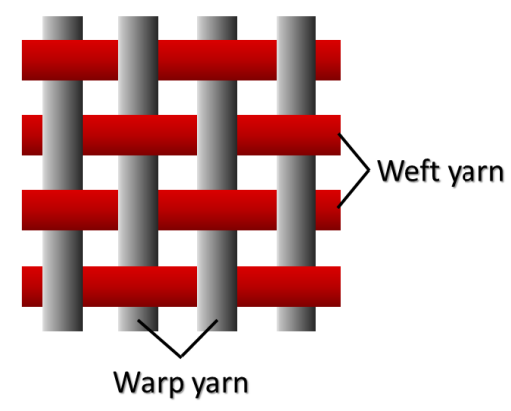

Figure 3. Definitions of warn yarn and weft yarn in weave fabric.

The two methods rely on different imaging systems and use different optical properties of the yarns. In the first method, the used property is the color. Color images of the fabric, presently a polyester Jacquard fabric made of red weft yarns and black warp yarns, are made with a flatbed scanner. The interest of this method is that image acquisition is easy to do, and the color image processing permitting to identify the respective positions of the yarns is rather simple. Its drawback is that the angle-dependent shine effects cannot be predicted. The second method, tested on satins made of polyester and metallo-plastic yarns, is based on the fact that polyester, a dielectric material, and metal-coated plastic do not reflect polarized light with the same polarization state ${ }^{10}$. Hence, by using collimated, polarized light and by placing a polarization filter in front of the camera, we can get the respective positions of the two types of yarns. Moreover, by making images under several incidence angles, the angle-dependent shine effects can be reproduced.

In the two methods, the color changing operations are based on very simple reflectance models, mainly consisting in adding an achromatic light component secularly reflected by the surface of the yarns to the chromatic light component internally scattered within the yarns. The multiple reflections between the yarns, as well as possible transparency effects, are ignored. It is assumed that a fabric woven with similar yarns (i.e. same chemical composition, same diameter, same spinning, same coating... and similar dyeing process but with different tint) will have similar gloss and shadow textures. 


\section{COLOR IMAGING METHOD}

The first method that we tested to localize the warp and weft yarns in the image, then to recolor them, is based on high resolution color imaging. It applies when the two yarns have sufficiently different intrinsic colors, such as the polyester Jacquard fabric woven with black warp yarns and red weft yarns shown in Figure 4. This fabric was digitalized at 2400 dpi in TIFF format, 24 bits, with an EPSON Perfection V700 PHOTO scanner. Our aim wass to virtually replace the red yarns with blue yarns in the scanned image.
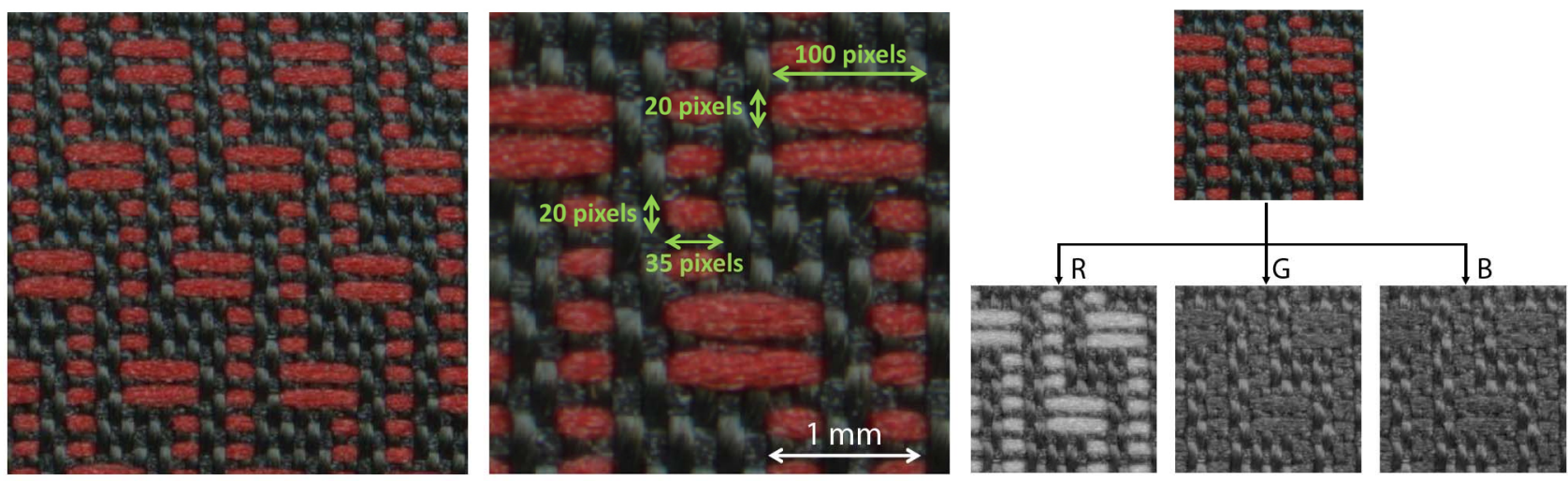

Figure 4. Left: the studied Jacquard fabric, scanned at 2400 dpi with a color-calibrated scanner. Middle: portion of image showing the size of yarns in pixels. Right: Red, Greed and Blue channels of the same portion of image.

\subsection{Maps of the yarns}

In order to modify the color of the red warp yarns, we had to identify the exact position of the pixels representing them in the image. In the Red channel image (Figure 4), these pixels look brighter than the rest of the image, but many pixels in the areas occupied by the black warp yarns have similarly bright grey level due to gloss. Simple thresholding on the Red channel was therefore insufficient. Instead, we used a more advanced algorithm based on the following steps:

1. The Green channel image was subtracted to the Red channel image; in the resulting grey level image, the pixels attributed to the red and black yarns belong to well distinct classes of grey levels.

2. This resulting grey level image was converted into a binary image by thresholding.

3. Noise was reduced by erosion then dilation ${ }^{11-13}$ in order to obtain smoother contours and remove isolated pixels in the areas corresponding to the warp yarns.

4. The resulting binary image was used as a mask on the original color image: only the red weft yarns are displayed, while the areas corresponding black warp yarns are set to black.

5. The complementary mask was applied on the original image, thus displaying only the black warp yarns.

Figure 5 shows the different images through this procedure (on a part of the original image).

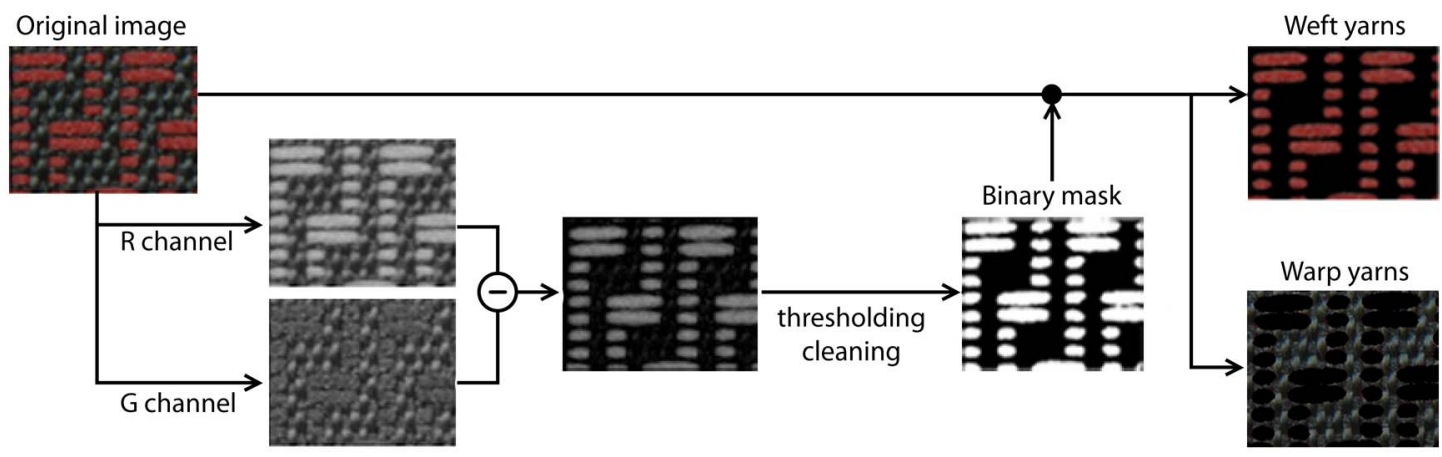

Figure 5. Main steps to localize the red weft yarns and black warp yarns in a portion of the scanned image of the fabric. 


\subsection{Changing the yarn colors}

We wanted to simulate the appearance of the fabric with blue yarns in place of the red yarns. We thus modified the color of the pixels of the previously obtained weft yarn image. Figure 6 shows pictures of a hank of the red yarns contained in the original fabric, as well as a hank of the blue ones that are used for the simulation. Both hanks were digitalized by using the same calibrated scanner as for the digitalization of the fabric. Once the colored yarns are woven with black yarns, their color looks darker, probably due to shadowing and transparency effects. This is noticeable by comparing the color of the hank of red yarns in Figure 6 and the color of the same red yarns in the fabric, in Figure 4.
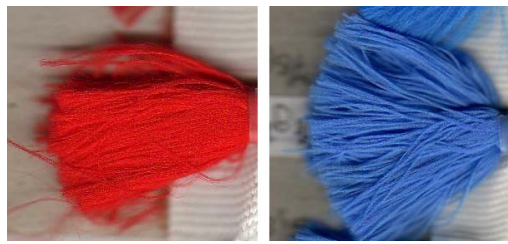

Figure 6. Hanks of the red yarns contained in the studied fabric (left), and of blue yarns used for the simulated fabric (right).

The color changing operation was based on a simple reflectance model for the weft yarns. We assumed that they are made of a uniform diffusing material with spectral reflectance $\rho(\lambda)$. In order to take the shadows into account, as well as the local irradiance variations and the transparency of the red yarns through which the black yarns are slightly visible, we weighted the reflectance in each pixel $(x, y)$ by an achromatic constant value $\sigma$ between 0 and 1 . Moreover, in order to account for the gloss, we added to each pixel an achromatic reflectance value $\gamma$. The reflectance of the yarn for each pixel was therefore modelled as

$$
R(x, y)=\gamma(x, y)+\sigma(x, y) \rho(\lambda)
$$

When replacing the red yarns with the blue ones, only the spectral reflectance $\rho(\lambda)$ was modified. If this spectral reflectance $\rho$ is in the same order of magnitude for the two colors of yarns, the color changing has no influence on the lightness of the total reflectance, but only on the hue and chroma. We also assumed that the constants $\sigma$ and $\gamma$ representing the gloss and shadow texture have an influence only on the lightness, not on the hue and chroma. The color changing operation is therefore performed in the CIE $\mathrm{L}^{*} \mathrm{C}^{*} \mathrm{~h}^{*}$ color space, after conversion of the RGB colors of the image into CIELAB colors by choosing a D65 illuminant ${ }^{14}$. The $\mathrm{C}^{*}$ and $\mathrm{h}^{*}$ color values are deduced from the $\mathrm{a}^{*}$ and $\mathrm{b}^{*}$ values thanks to the following relations:

$$
C^{*}=\sqrt{a^{* 2}+b^{* 2}} \quad \text { and } \quad h^{*}=\arctan \left(b^{*} / a *\right)
$$

From the scanned images of the hanks of yarns, we computed the average $h^{*}$ value for the red yarns, $h_{r}$, and of the blue yarns, $h_{b}$. Then, in the weft yarn image of the fabric, we shifted the $h^{*}$ value of each pixel by an angle $h_{b}-h_{r}$. The chroma of the two yarns being comparable, it was not modified. The lightness remained also unchanged, in order to conserve the gloss and shadow texture. Finally, the weft yarn image and the warp yarn image were added in order to obtain the preview of the fabric with blue yarns. The original and modified images are shown in Figure 7, for two types of Jacquard weavings.
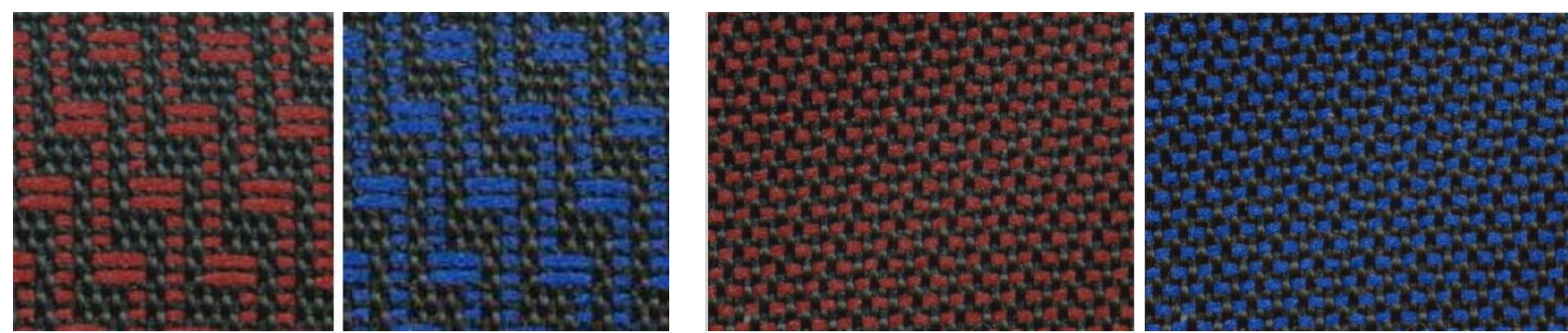

Figure 7. Original scanned images of fabrics with red weft yarns and simulated images of the fabric with blue weft yarns for two different Jacquard weavings. 
In order to estimate the image quality of the simulated images of fabrics, we presented the original images of fabric with red yarns and the simulated images of fabric with blue yarns to a panel of 22 persons who didn't previously see the real fabric, and asked them which one is the original. Twelve of them thought that the original fabric is the red one, and ten the blue one, which tends to show that the simulated image looks as plausible as the original image and that the color changing operation does not induce perceptible decrease of image quality.

\subsection{Multiscale preview}

The simulated image obtained so far is not representative of the color that would be seen far from the fabric, especially at a distance where the weaving texture is not visible and only an average color is perceived. These changes of appearance as a function of the viewing distance can be easily simulated by decreasing the resolution of the simulated image by bilinear downsampling in a rate corresponding to the viewing distance. An example is shown in Figure 8.
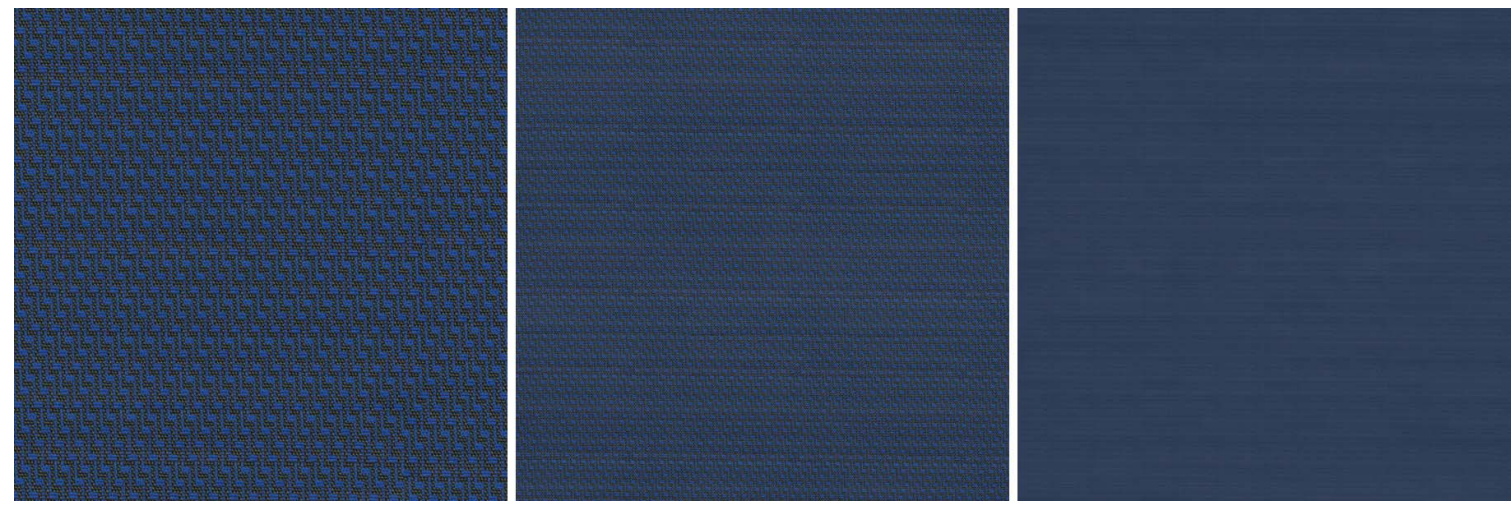

Figure 8. Simulated fabric at real scale (left), and as would be seen from a double distance (middle) and a distance multiplied by 16 (right).

\subsection{Discussion on the color imaging method}

The color imaging method has the advantage of simplicity: a simple scanner is necessary as measurement device, and the image processing is easy to do when the yarns in the original fabric are sufficiently different and the replacement color of yarn has similar chroma and reflectance has the original one. However, this method has several limitations. The first one comes from the measurement device: since it is based on a fixed illumination and observation geometry, it enables simulations only for this geometry. The angle-dependent shine or shadow effects cannot be predicted. This problem can be solved by replacing the scanner by a photo camera and a rotatable collimated light source, and making several images under different illumination angle. The color changing algorithm would thus be applied on the different images.

Regarding the color changing algorithm, it has also several limitations. If one of the original yarns is too bright, e.g. white, the color picture may be saturated and reflectance information may be missed. Secondly, the method is operative if the original and replacement yarns have reflectances with similar order of magnitude: the shadows and gloss texture may be significantly different on white yarns than on darker yarns. In order to solve this problem, by assuming that the gloss and shadow texture is mainly contained in the $L^{*}$ channel of the image represented in the CIELAB color space, we can study from several fabrics how the histogram of the $L^{*}$ values varies according to the intrinsic reflectance of the yarns, and find a transformation law that convert the $L^{*}$ values of the original yarns into $L^{*}$ values of the replacement

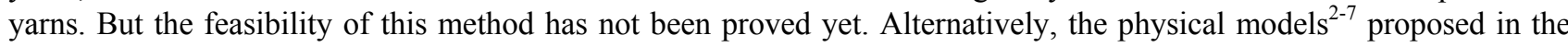
literature for predicting the color of blends of fibres could be used.

\section{POLARIZED IMAGING METHOD}

The second method that we tested to localize the warp and weft yarns is based on polarized imaging. This technique is especially suitable with blends of metallic and polymer yarns, since metals and dielectrics (such as polymers) do not reflect linearly polarized light in the same way: the light reflected by a dielectric remains polarized and can be blocked by a polarizing filter, whereas the light reflected by a metal has generally an elliptical polarization and is only partially 
blocked by the polarizing filter ${ }^{15}$. We propose to illustrate this effect with pieces of satin woven with warp yarns in polyester (polyester is a dielectric material) and weft yarns in metal-coated plastic.

\subsection{Imaging system}

The imaging system is based on the SONY XCD-X710 IEEE 1394 grey-level camera, preferred to a RGB camera for higher sensitivity and higher resolution, and the HL-2000-HP 20W halogen light source from Edmund Optics with optical fiber. The output of the optical fiber is placed at the focus of a lens in order to create a collimated light beam of 2 $\mathrm{cm}$ diameter. A polarizing filter is placed after the lens in order to linearly polarize the incident light, and colorimetric filters may be added in order to make color images (RGB images are made in three steps, by making one grey level image with each filter). The whole illumination system is mounted on a rotary plate which enables variations of the incidence angle. Angular, polarized and color imaging is therefore permitted with this illumination system.
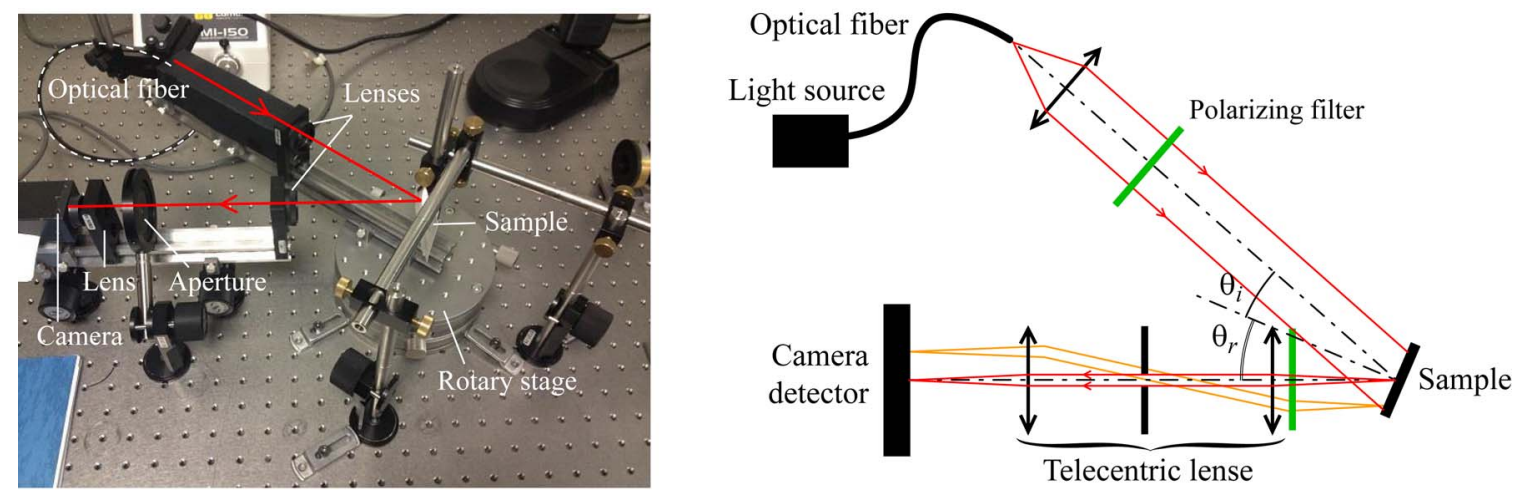

Figure 9. Picture and drawing of the angular, polarized imaging system.

The sample is placed at the center of rotation of the incident beam. The camera is fixed and views the sample at $20^{\circ}$ of its normal. It is preceded by a second polarizing filter and a telecentric lens made of two lenses and an aperture: the aperture and the sample are in the focus planes of the first lens, and the aperture and the camera detector in the focus planes of the second lens. Thanks to the telecentric lens, every pixel on the detector views the sample under the same angle exactly ${ }^{16}$. Schematic view and picture of the imaging system are shown in Figure 9.

Measurements were performed on two pieces of satin at different incident angles from $0^{\circ}$ to $60^{\circ}$ in steps of $5^{\circ}$. The first satin, achromatic, was woven with black polyester and silvered metal-coated plastic yarns. The second one, colored, was woven in the same conditions with dark red polyester and golden metal-coated plastic yarns. The achromatic satin was observed in grey-level. The grey-level images were converted into RGB images where each channel reproduces the greylevel image. For the colored satin, we introduced red, green and blue colorimetric filters from Johnson Cousins, and made one grey-level image with each of them. Similar color image was made with the same filters and the same lighting of a perfectly white diffuser of albedo close to 1 from Labsphere in order to perform a white balance. Our experiment aimed at coloring the achromatic satin in order to get similar appearance as the colored one.

\subsection{Maps of the yarns and changing of color}

The map of the yarns was performed from the achromatic satin. For each incident angle, two images were made. For the first image, the polarizing filter in front of the camera was rotated in order to block all the light reflected by the polyester yarns (cross polarization configuration). This image corresponds to the gloss map due to the metallic yarns only. For the second image, the polarizing filter was rotated by $90^{\circ}$ (parallel polarization configuration) and the gloss on the polyester yarns appeared. Since the metallic yarns have very similar appearance in the two images, subtracting one from the other one yields a map of the polyester yarns only. Note that perfect registration of the two images is necessary; otherwise, part of the light reflected by the metallic yarns would remain visible. Figure 10 shows the images made in cross polarization and polarization as well as the difference between the two images for an incident angle of light of $20^{\circ}$ and an observation angle of $20^{\circ}$. 

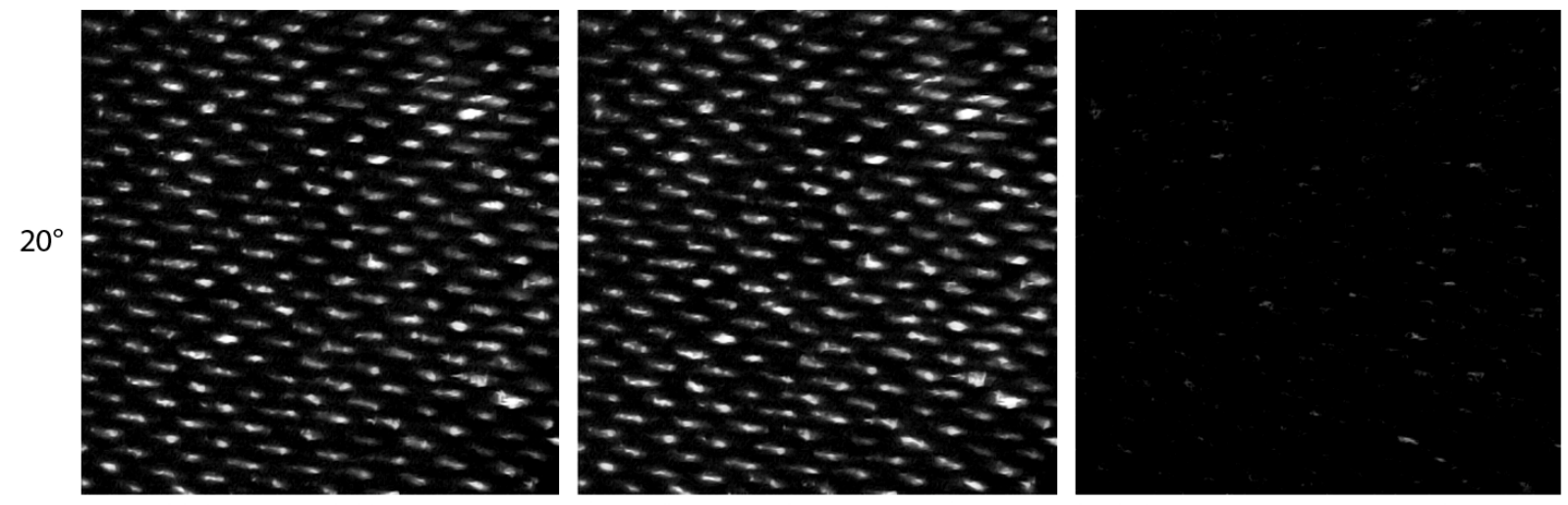

$30^{\circ}$
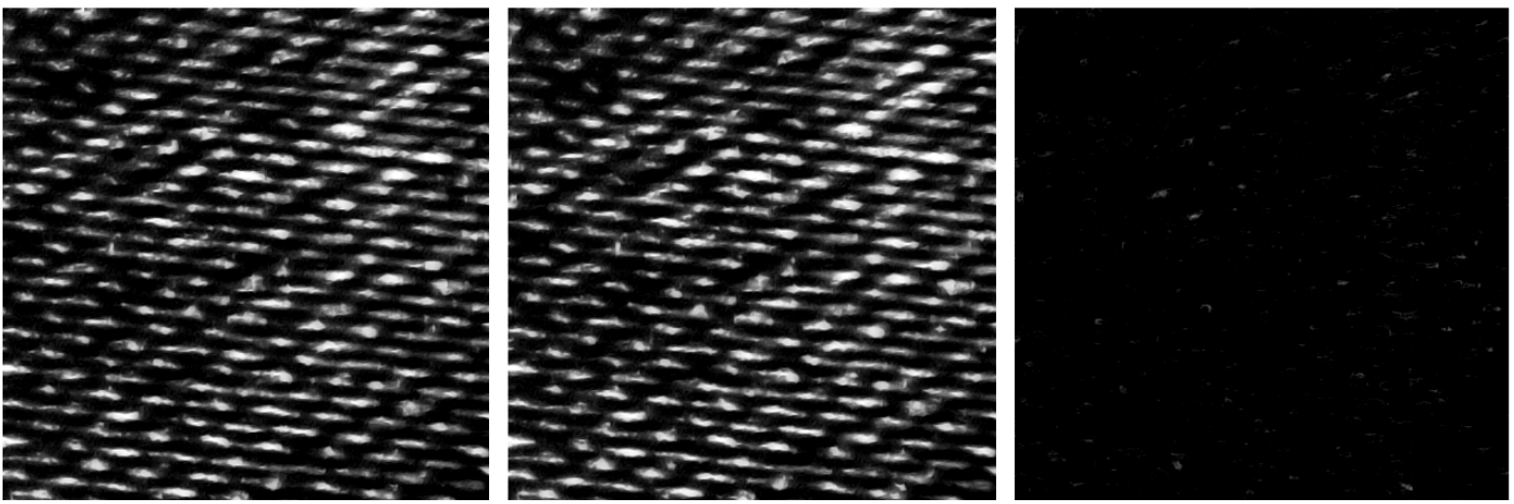

Figure 10. Images of the fabric made under collimated illumination at $20^{\circ}$ (top row) and $30^{\circ}$ (bottom row) from the normal, and observation at $20^{\circ}$, either in cross polarization configuration (left) or parallel polarization configuration (middle), and difference between the polarized images (right). The left-most images show the gloss maps of the metal coated plastic yarns and the right-most images the gloss maps of the polyester yards.

In the fabric selected for our experiments, we could assume that the two yarns have no diffuse reflectance and achromatic specular reflectance. It was the case for the black polyester yarns, whose intrinsic reflectance is assumed to be zero. It was also the case for the silvered metal-coated plastic yarns, achromatic and almost perfectly specular even though a twinkling effect is observed when varying the orientation of the lighting or the viewing position, due to their spinning. The light signal captured by the camera therefore corresponded, almost exclusively, to gloss. As no color information was needed, the grey level camera was selected for better resolution and sensitivity.

The changing of yarn color was based on the following assumptions. Colored polyester yarns reflect the same achromatic gloss as the black ones, and additionally reflect a Lambertian flux with a given spectral power distribution (SPD). For the black yarns, this Lambertian reflectance was assumed to be zero. Hence, we added to each pixel of the gloss map measured on the black yarns a RGB value $\left(r_{d}, g_{d}, b_{d}\right)$ corresponding to the SPD of the colored yarn's Lambertian reflectance. Regarding the colored metal-coated plastic yarns, we assumed that they reflect similar gloss as the achromatic ones but with a specific SPD, independent of the incident angle of light. The Red, Green and Blue channels of the gloss map measured on the silvered yarns were respectively multiplied by constant values $r_{s}, g_{s}$, and $b_{s}$ corresponding to the SPD of the colored gloss. Finally, the colored gloss maps of the polyester and the metallic yarns were added. The values $r_{d}, g_{d}, b_{d}$ and $r_{s}, g_{s}, b_{s}$ were selected in order to match the color appearance of the images made on the red/gold satin. The simulated image and the measured one corresponding to the incident angle of $20^{\circ}$ and $30^{\circ}$ are shown in Figure 11. Even though it is difficult to quantify the deviation between the two images, we can notice their visual similarity. By applying this method on the images made at different incident angles and displaying them successively, the twinkling effect due to metallic yarns is reproduced. 

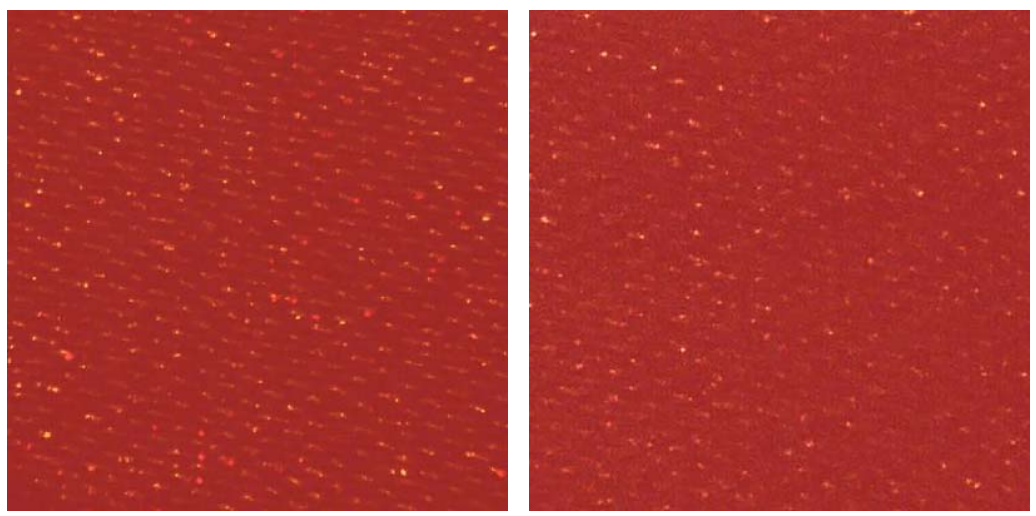

Figure 11. The image of a red/gold satin, obtained by coloration of the measured black/silvered satin (left) is compared to the measured image of a real fabric (right).

\subsection{Discussion on the polarized imaging method}

The polarized imaging method is especially suitable for blends of dielectric and metallic yarns, which are frequently used in the packaging industry (see Figure 2). In the example treated here, the simple reflectance model that we used seems to be sufficiently accurate to provide good visual preview on a computer screen either in static mode (single incident angle) or dynamic mode (sequence of images made under different incident angles, showing the twinkling effect). For the measurements, an achromatic fabric sample is recommended because it eases considerably the virtual coloration process, which is simpler than the one used in the color imaging method. Fortunately, achromatic samples are rather common in the textile industry. The color values applied in the coloration method could be easily measured on hanks of yarns and stored in a database. In this method, measurements are slightly more constraining than in the color imaging method since two images are necessary with a rotation of the polarizing filter.

It seems also necessary to discuss the limitations inherent in imaging systems for reproducing the appearance of surface containing such highly specular components as the metallic yarns. The dynamic of most imaging systems is often insufficient to cover the range of luminances reflected by theses surfaces. The image is thus saturated in the pixels impacted by thin high-luminance rays. The aperture of the lens and the resolution of the detection array may also have a significant impact on the gloss texture image. Modelling the perception of visual texture and gloss in real objects and digital images would represent a high benefit ${ }^{17}$. As a consequence, it is difficult to simulate the rendering of the fabric at a larger viewing distance. Applying the bilinear downsampling method proposed in Section 2 for the polyester fabric, whose contrast gloss is rather low, would strongly attenuate the contrast of the gloss texture here, whereas we rather expect that this contrast remains constant while increasing the viewing distance. Further investigation would be necessary on this question.

\section{CONCLUSIONS}

For a more realistic simulation of the appearance of fabrics with different yarn tones, we suppose that the shadow and gloss texture must be reliably reproduced and unaffected by the yarn color changing process. We proposed two methods satisfying this constraint, based on either color imaging or polarized imaging according to the type of fabric: the color imaging method is suitable for fabrics woven with similar yarns having different colors, such as Jacquard weave fabrics, whereas the polarized imaging method is restricted to blends of dielectric and metallic yarns. The fact that the gloss texture is conserved in the simulated images certainly represents a gain in rendering realism in comparison to the average reflectance prediction models. The use of a rotating light source is interesting to show and reproduce the gonioapparent effects displayed by most textiles. It was tested with the polarized image method because the angle-dependent rendering was more striking, but it could have been also used with the color imaging method presented in Section 2, even though illuminating a large area with collimated light is often a practical issue. The first experiments reported in this paper simply show the feasibility of modifying selectively the color of yarns, and obtaining simulated images that have as good quality as the original images. Further improvements would be necessary to simulate the visual rendering under different types of lighting, under different viewing distances and at different viewing angles. The next challenge is also to be able 
to simulate the appearance of such fabrics on non flat surfaces, which is the case in most practical situations, from images made on a flat area.

\section{ACKNOWLEDGMENTS}

This work was supported by the French National Research Agency (ANR) within the program "Investissements d'Avenir" (ANR-11-IDEX-0007), in the framework of the LABEX MANUTECH-SISE (ANR-10-LABX-0075) of Université de Lyon.

\section{REFERENCES}

[1] Stearns, E.I., Noechel, F., "Spectrophotometric prediction of color wool blends," American Dyestuff Reporter 33(9) 177-180 (1944).

[2] Philips-Invernizzi, B., Dupont, D., Cazé, C. "Formulation of colored fiber blends from Friele's theoretical model," Color Res. Appl., 27: 191-198 (2002).

[3] Rong, L. I. , Feng, G. U., "Tristimulus algorithm of colour matching for precoloured fibre blends based on the Stearns-Noechel model," Coloration Technology 122, 74-81 (2006).

[4] Davidson, H. R., Taylor, M., "Prediction of the Color of Fiber Blends," J. Opt. Soc. Am. 55, 96-98 (1965).

[5] Burlone, D. A., "Theoretical and practical aspects of selected fiber-blend color-formulation functions," Color Res. Appl. 9, 213-219 (1984).

[6] Amirshahi, S.H., Pailthorpe, M.T., "An Algorithm for Optimizing Color Prediction in Blends," Textile Research Journal 65, 632-637 (1995).

[7] Mathur, K., Hinks, D., Seyam, AF. M., Donaldson, R.A., "Towards Automation of Color/Weave Selection in Jacquard Design: Model Verification," Color Res. Appl., 34: 225-232 (2009).

[8] Shidanshidi, H., Naghdy, F., Naghdy, G., Conroy, D.W., "3D Geometric Modelling of Hand-Hoven Textile," IS\&T/SPIE 20th Annual Symposium Electronic Imaging Science and Technology, SPIE 6813, Image Processing: Machine Vision Applications, 68130B-1-68130B-10 (2008).

[9] Shidanshidi, H., Naghdy, F., Naghdy, G., Conroy, D.W., "3D Geometric and Haptic Modeling of Hand-Woven Textile Artifacts" in Cultural Computing 333, Ed. Nakatsu, R., Tosa, N., Naghdy, F., Wong, K.W., Codognet, P., Springer, pp. 87-100 (2010)

[10] Wolff, L. B., "Classification of Material Surfaces Using the Polarization of Specular Highlights," Columbia University, Computer Science department (1988) http://academiccommons.columbia.edu/catalog/ac\%3A142380.

[11] Serra, J. [Image Analysis and Mathematical Morphology] Academic Press, New-York (1982).

[12] Serra, J. [Image Analysis and Mathematical Morphology, Part II: Theoretical Advances] Academic Press, London, (1988).

[13] Soille, P. [Morphological Image Analysis], Springer-Verlag, Berlin (1999).

[14] Sharma, G. [Digital Color Imaging Handbook] CRC Press, (2002).

[15] Born, M., Wolf, E. [Principle of Optics], 7th ed., Pergamon, p. 741 (1999).

[16] Lequime, M., Zerrad, M., Deumié, C., Amra, C. "A goniometric light scattering instrument with high resolution imaging," Optics Communications 282, 1265-1273 (2009).

[17] Ferwerda, J., Fores, A., Tastl, I., Recker, J. "Perceiving gloss in surfaces and images," J. Vision 14(10) article 453 (2014). 